\title{
Mechanism of Rotational Hysteresis Energy in Sm-Fe-N Permanent Magnets
}

\author{
P. PAWLIK, J.J. WysŁocki* AND K. DzILIŃSKI \\ Institute of Physics, Technical University of Częstochowa \\ Al. Armii Krajowej 19, 42-200 Częstochowa, Poland
}

(Received August 24, 2001)

\begin{abstract}
Investigations were carried out on $\mathrm{Sm}-\mathrm{Fe}-\mathrm{N}$ permanent magnet produced by the reactive diffusion method with different grain sizes (from 8.6 to $0.97 \mu \mathrm{m}$ ). The rotational hysteresis energy has been measured as a function of the applied field. The proposed model of rotational hysteresis energy is in good agreement with the experimental results. It is shown that the magnetization reversal process in $\mathrm{Sm}-\mathrm{Fe}-\mathrm{N}$ magnet is controlled by the nucleation of reversed domains.
\end{abstract}

PACS numbers: 75.30.-m, 75.60.-d

\section{Introduction}

The investigations of $\mathrm{Sm}-\mathrm{Fe}-\mathrm{N}$ permanent magnet carried out so far were focused on the formation of permanent magnet properties [1-6], thermal decomposition of the primary hard magnetic $\mathrm{Sm}_{2} \mathrm{Fe}_{17} \mathrm{~N}_{3}$ phase [7] or microstructure and domain structure observations [8-10]. This magnet has become, since its discovery in 1990 [1], one of the most promising modern hard magnetic materials, which exhibits outstanding magnetic properties and it is suitable for various applications, e.g., in hysteresis motors, magnetic drives and clutches. However, there is a lack of reports on rotational hysteresis energy in this material. This is rather a surprising situation, since a large number of machines and devices exists in which $\mathrm{Sm}-\mathrm{Fe}-\mathrm{N}$ magnets are subjected to rotating magnetic fields. Moreover, as was shown in [11-14], significant information about the magnetization reversal process may be retrieved from the investigations of the rotational hysteresis energy $W_{\mathrm{r}}$ determined from the torque curves $T$ in a rotating external magnetic field [15].

Therefore, the main aim of this paper is to determine the mechanism of rotational hysteresis energy in $\mathrm{Sm}-\mathrm{Fe}-\mathrm{N}$ permanent magnets.

*corresponding author; e-mail: wyslocki@mim.pcz.czest.pl 


\section{Material and experimental methods}

Measurements were performed on $\mathrm{Sm}-\mathrm{Fe}-\mathrm{N}$ magnets produced by the reactive diffusion method [16]. The production process consists of annealing of Sm and $\mathrm{Fe}$ powder mixture under pressure of $100 \mathrm{MPa}$ at a temperature of $1320 \mathrm{~K}$ (i.e. below the melting temperature of components) for $5 \mathrm{~h}$. As a result of solid state diffusion a relatively isotropic crystalline structure is achieved. After initial milling for $20 \mathrm{~min}$ the powders are nitrided at $750 \mathrm{~K}$ for $3 \mathrm{~h}$. The final milling of powders is used to get a required grain size. Then the powders together with thermosetting plastic are pressed $(100 \mathrm{MPa})$ in aligning magnetic field in order to obtain magnets that exhibit anisotropic magnetic properties. This production method is much simpler than the conventional melting of components, because it does not require high temperature heat treatment and consequently samples are less exposed on oxidation or sublimation effects during manufacturing process.

The investigations were carried out on samples after following final milling time: $2,6,12,24$, and $48 \mathrm{~h}$. Diameters of the obtained powders are: $8.6 \mu \mathrm{m}, 6.6 \mu \mathrm{m}$, $3.3 \mu \mathrm{m}, 2.2 \mu \mathrm{m}, 1.6 \mu \mathrm{m}$, and $0.97 \mu \mathrm{m}$, respectively.

Using an X-ray microanalyzer as well as X-ray diffractometer with Co $K_{\alpha}$ radiation, qualitative analysis of phase structure was performed. Furthermore, using Mössbauer spectrometer with ${ }^{57} \mathrm{Co}$ isotope in rhodium matrix and constant acceleration, the quantitative analysis of examined samples was carried out. Measurements of Mössbauer spectra were performed at room temperature.

Anisotropy constants $K_{1}$ and $K_{2}$ were determined from the Fourier analysis of the torque curves in the field close to $2 \mathrm{MA} / \mathrm{m}$, and then using extrapolation of the results to infinitely high magnetic field $1 / H$. Measurements of the torque curves were performed using torsion magnetometer for different values of external magnetic field up to $H=2 \mathrm{MA} / \mathrm{m}$.

\section{Torque curves and rotational hysteresis energy}

The rotational hysteresis energy $W_{\mathrm{r}}$, i.e. the energy necessary for a $360^{\circ}$ turn of the magnetic material in constant magnetic field, can be experimentally determined as the algebraic sum of the areas determined by the positive and negative parts of the torque curve and the $\theta$ axis, measured at clockwise (CW) rotation of the external magnetic field. However, if subsequently the field is rotated through $360^{\circ}$ in the anticlockwise direction (ACW), the two torque curves (corresponding to $\mathrm{CW}$ and $\mathrm{ACW}$ rotations of the magnetic field) enclose an area which is equal to twice the value of rotational hysteresis energy:

$$
W_{\mathrm{r}}=\frac{1}{2}\left[\int_{0}^{2 \pi} T_{\mathrm{CW}}(\Theta) \mathrm{d} \Theta-\int_{0}^{2 \pi} T_{\mathrm{ACW}}(\Theta) \mathrm{d} \Theta\right],
$$

where $\Theta$ is the angle between the easy magnetization axis and the direction of the 


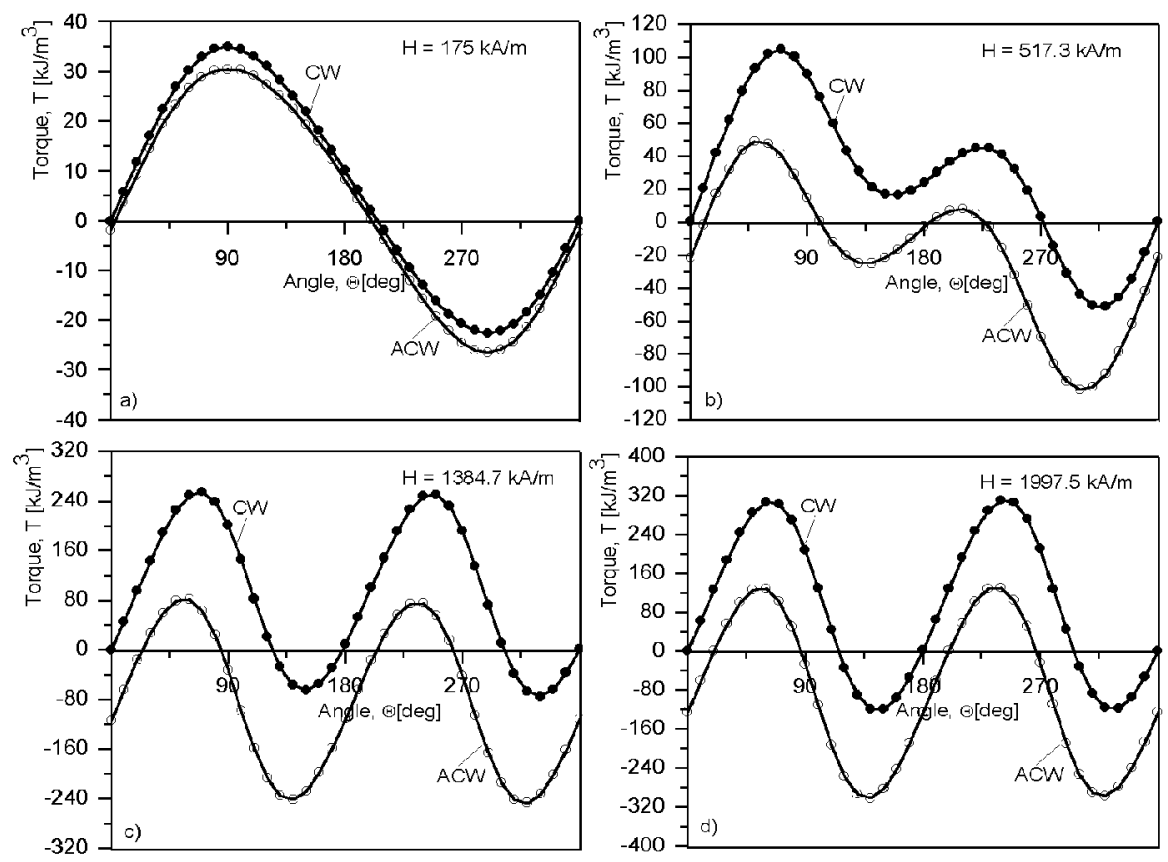

Fig. 1. The examples of torque curves of the $\mathrm{Sm}-\mathrm{Fe}-\mathrm{N}$ magnet with a $2.2 \mu \mathrm{m}$ grain diameter for several values of the external magnetic field $H$ : (a) $175 \mathrm{kA} / \mathrm{m}$, (b) $517.3 \mathrm{kA} / \mathrm{m}$, (c) $1384.7 \mathrm{kA} / \mathrm{m}$, (d) $1997.5 \mathrm{kA} / \mathrm{m}$.

external magnetic field, $T_{\mathrm{CW}}(\Theta), T_{\mathrm{ACW}}(\Theta)$ denote the torques measured, respectively, at clockwise and anticlockwise rotation of the external magnetic field. As an example the torque curves $T$ vs. $\Theta$ angle for the $\mathrm{Sm}-\mathrm{Fe}-\mathrm{N}$ magnet with a grain diameter of $2.2 \mu \mathrm{m}$ are presented in Fig. 1. The curves were measured for different values of external magnetic field. The torque curves for $\mathrm{CW}$ and $\mathrm{ACW}$ rotations are identical when the rotational hysteresis energy $W_{\mathrm{r}}$ is equal to zero. This can occur if the external magnetic field is too low and energy losses with rotational hysteresis are still absent (the $T(\Theta)$ curve is described by a $\sin \Theta$ function), or if the field is sufficiently strong to saturate the sample and energy losses are no longer present $(T(\Theta)$ curve is described by a $\sin 2 \Theta$ function). However, the external magnetic field used in the experiment is insufficient to saturate the examined samples, so that there is still small rotational hysteresis losses (Fig. 1d).

In Fig. 2 the dependence of the rotational hysteresis energy normalized by the anisotropy constant $W_{\mathrm{r}} / K_{1}$ on the reduced applied magnetic field $h=H / H_{\mathrm{a}}$ (where $H_{\mathrm{a}}=7500 \mathrm{kA} / \mathrm{m}$ is the anisotropy field) for $\mathrm{Sm}-\mathrm{Fe}-\mathrm{N}$ magnets with grain diameters: $0.97 \mu \mathrm{m}, 1.6 \mu \mathrm{m}, 2.2 \mu \mathrm{m}, 3.3 \mu \mathrm{m}$, and $8.6 \mu \mathrm{m}$, is presented. These curves were determined from the torque curves analysis, using Eq. (1). The measurements were carried out for external magnetic field within the range from 0 to 


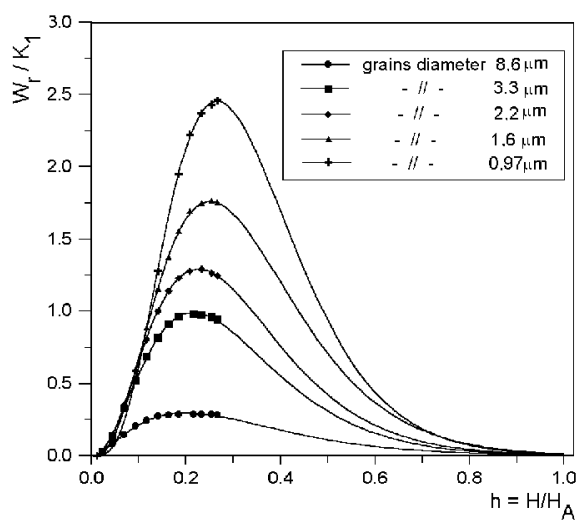

Fig. 2. Rotational hysteresis energy $W_{\mathrm{r}} / K_{1}$ dependence on the reduced magnetic field $h=H / H_{\mathrm{a}}$ for the $\mathrm{Sm}-\mathrm{Fe}-\mathrm{N}$ magnet with different grain diameters.

$2.0 \mathrm{MA} / \mathrm{m}(h=0.27)$. From Fig. 2 it results that the rotational hysteresis energy begins in low fields (below $h=0.05$ ) and reaches a maximum value depending on the grain diameter. For example, the magnet with a $8.6 \mu \mathrm{m}$ grain diameter reaches the maximum $\left(W_{\mathrm{r}}\right)_{\max } / K_{1}=0.29$ for the reduced magnetic field $h=0.2$ and for the magnet with a $1.6 \mu \mathrm{m}$ grain diameter $\left(W_{\mathrm{r}}\right)_{\max } / K_{1}=1.75$ at $h=0.25$. For the magnets with a smaller grain diameter $(0.97 \mu \mathrm{m})$, external magnetic field used for the experiment was insufficient to reach the maximum value of the rotational hysteresis energy $\left(W_{\mathrm{r}}\right)_{\max }$. Therefore, the maximum value of rotational hysteresis energy $\left(W_{\mathrm{r}}\right)_{\max }=1092 \mathrm{~kJ} / \mathrm{m}^{3}\left(\left(W_{\mathrm{r}}\right)_{\max } / K_{1}=2.48\right)$ was determined using extrapolation formula [15]. This maximum value corresponds to the reduced magnetic field $h=0.28$. Furthermore, a shift of maximum values of $\left(W_{\mathrm{r}}\right)_{\max } / K_{1}$ to the higher values of the external magnetic field (from $h=0.2$ to 0.28 ) with decreasing the grain diameter, is observed. The results of this paper are not accessible to an interpretation based on the single-domain particle theory of Stoner-Wohlfarth [17] (coherent rotation model), Jacobs-Bean theory [18] (incoherent rotation model, referred to as fanning), or the more general Shtrikman-Treves theory [19] for infinitely long cylindrical single domain particles of various radii taking into account the influence of incoherent mechanisms of magnetization reversal (referred to as curling). In these models the rotational hysteresis energy emerges jumpwise and reaches its maximum value $\left(W_{\mathrm{r}}\right)_{\max }$ in a field of $h=0.5$ in Stoner-Wohlfarth approach, or in a field $h=0.33$ in that of Jacobs-Bean and decreases to zero for $h=1$. In Shtrikman-Treves theory they are dependent on the reduced radius $S=D / D_{0}$ (where $D$ is the radius of the cylinder and $D_{0}$ - a characteristic radius [19]). When $S \ll 1$, the change in magnetization occurs by coherent rotations of the magnetization vector and, when $S>1$, by incoherent mechanism. For the first two of the above models, the ratio $\left(W_{\mathrm{r}}\right)_{\max } / K_{1}=2.67$ whereas for the examined Sm-Fe-N magnet $\left(W_{\mathrm{r}}\right)_{\max } / K_{1}$ varies from 0.29 (in a field of $h=0.2$ for a grain diameter of $8.6 \mu \mathrm{m}$ ) to 2.48 (in a field of $h=0.28$ for a grain diameter 
of $0.97 \mu \mathrm{m}$ ). It means that for the examined magnets with smaller grains, the reduced field $h$ corresponding to the maximum value of rotational hysteresis energy $\left(W_{\mathrm{r}}\right)_{\max }$ approaches the value of $h=0.33$, characteristic of Jacobs-Bean theory. However, the $\left(W_{\mathrm{r}}\right)_{\max } / K_{1}$ values correspond to the $S$ parameter varying from 2.0 to 2.4 (depending on the grain size), which according to Shtrikman-Treves theory provides evidence for an incoherent rotation.

\section{Comparison of experimental results with theoretical description}

A good fitting of the experimental data of the rotational hysteresis energy can be achieved using the following formula:

$$
\frac{W_{\mathrm{r}}}{K_{1}}=A\left(\frac{H}{H_{\mathrm{a}}}\right)^{\beta} \exp \left(-B \frac{H}{H_{\mathrm{a}}}\right),
$$

where $A, B, \beta$ are the extrapolation parameters. This formula is the generalization of the equation proposed by Bryukhatov and Grintshchar [20] (with $\beta=1$ ) and used in Refs. [21, 22] to describe the rotational hysteresis energy. In the paper we have obtained a better approximation of the theoretical curves to experimental data by introducing the coefficient $\beta>1$. In Table the values of $A, B$, and $\beta$ coefficients for different grain diameters are listed. With a decrease in grains diameter, an increase in $A, B$, and $\beta$ values is observed.

The origin of the rotational hysteresis energy is usually derived from the fact that the magnetization vector $M_{\mathrm{s}}$ does not keep up with the rotations of the external magnetic field. Akulov [23] proved that the maximum of rotational hysteresis energy corresponds to the transition from the spins interaction with a crystalline structure to their interactions with external magnetic field. Such transition in rotating external magnetic field may occur jumpwise, just like in Barkhausen effect, where jump-like reorientation of domain structure is also caused by rotating external magnetic field. This reorientation may also occur by separation of magnetic moments of domains into the local magnetic moments (spin complexes), which differently interact with the crystalline structure. The local moments may become the nuclei of reversed domains, which form in the areas of reduced anisotropy energy, e.g. grain boundaries, phase boundaries or inclusions of different phases in the area of magnetic phase. Therefore, the rotational hysteresis for a given value of the external magnetic field is a dynamic equilibrium state between magnetic moments of domains that strongly interact with the crystalline structure and local magnetic moments that weakly interact with the crystalline structure and which change the direction of magnetization. They form a mean angle $\Phi$ with the external magnetic field.

According to the model introduced by Bryukhatov and Grintshchar [20], the number of local magnetic moments, which follow this separation process in a time-defined period, determines the amount of irreversible magnetization reversal and, at the same time, is intermediately correlated with the rotational hysteresis 
energy $W_{\mathrm{r}}$. Furthermore, Boltzmann distribution of local magnetic moments was assumed:

$$
N_{1}=N \exp \left(-\frac{\Delta E}{N k T}\right),
$$

where $N$ is the number of all local magnetic moments in the volume unit $\left(1 \mathrm{~m}^{3}\right)$ being in a metastable state under the effect of external magnetic field; $N_{1}$ is the number of local magnetic moments possessing a mean value of activation energy $\Delta E$, which is necessary to overcome the interactions with a crystalline structure for a given value of the rotating external magnetic field and a mean value of $\Phi$ angle; $k$ is the Boltzmann coefficient; $T$ is temperature. In particular, $\Delta E$ in Eq. (3) describes the increase in potential energy of spin complexes, from $H=0$ state to the state with external magnetic field. For the following calculations the total free energy of the system in external magnetic field is assumed as

$$
E_{t}=E_{\mathrm{e}}+E_{\mathrm{a}}+E_{H}+E_{\mathrm{d}}
$$

where:

$-E_{\mathrm{e}}$ - exchange energy is described as

$$
E_{\mathrm{e}}=A\left(\frac{\mathrm{d} \Psi}{\mathrm{d} z}\right)^{2}
$$

$A$ - exchange constant; $\Psi$ - the angle between the easy direction of magnetization and the saturation magnetization vector $M_{\mathrm{s}}$;

- $E_{\mathrm{a}}$ - anisotropy energy equal to

$$
E_{\mathrm{a}}=K_{1} \sin ^{2} \Psi+K_{2} \sin ^{4} \Psi,
$$

where $K_{1}$ and $K_{2}$ - magnetocrystalline anisotropy constants;

$-E_{H}$ - energy of external magnetic field

$$
E_{H}=-\mu_{0} H M_{\mathrm{s}} \cos \Phi,
$$

where $\Phi$ - the angle between the saturation magnetization vector $M_{\mathrm{s}}$ and the direction of external magnetic field;

$-E_{\mathrm{d}}$ demagnetization energy

$$
E_{\mathrm{d}}=\frac{1}{2} \mu_{0} D M^{2}
$$

where $D$ - demagnetization coefficient, which is equal to 1 for the disc-shaped sample in the direction perpendicular to the disc surface and $M$ - magnetization vector.

The energy of external magnetic field is negative and its absolute value is greater than other positive contributions of total free energy [15, 24]. Therefore, these positive contributions might be considered as the factors that decrease the absolute value of total free energy. Taking into account the above equations the distribution (3) can be modified as 


$$
N_{1}=N \exp \left(\frac{-\mu_{0} M_{\mathrm{s}} H \cos \Phi+\frac{1}{2} \mu_{0} D M^{2}+E^{\prime}}{N k T}\right),
$$

where $E^{\prime}=E_{\mathrm{a}}+E_{\mathrm{e}}$.

In order to determine the rotational hysteresis energy $W_{\mathrm{r}}$ the following formula is used:

$$
W_{\mathrm{r}}=\int_{0}^{2 \pi} T(\Theta) \mathrm{d} \Theta
$$

where $\Theta$ - the angle between the easy direction of magnetization and the external magnetic field vector, and $T$ denotes the torque defined as

$$
T=\frac{N_{1}}{N} \mu_{0} H M_{\mathrm{s}} \sin \Phi,
$$

where $\Phi$ - the angle between magnetization vector and external magnetic field $H$; the ratio $N_{1} / N$ means the relative number of local magnetic moments that interact with the crystalline structure.

Because even for small values of external magnetic field the magnetization vector rotates towards the direction of magnetic field, the mean value of $\Phi$ angle was assumed. Using Boltzmann distribution (5) and substituting (7) to (6) as well as dividing by $K_{1}$, the relation describing normalized rotational hysteresis energy $W_{\mathrm{r}} / K_{1}$ was achieved as

$$
\begin{aligned}
\frac{W_{\mathrm{r}}}{K_{1}} & =\frac{\mu_{0} M_{\mathrm{s}} H}{K_{1}} \sin \Phi \exp \left(\frac{\mu_{0} D M^{2}}{2 N k T}\right) \\
& \times \exp \left(-\frac{\mu_{0} M_{\mathrm{s}} H \cos \Phi}{N k T}\right) \int_{0}^{2 \pi} \exp \left(\frac{E^{\prime}}{N k T}\right) \mathrm{d} \Theta .
\end{aligned}
$$

Regarding the fact that the magnetization $M$ depends on the external magnetic field the corresponding function can be written as [15]

$$
M(H)=M_{\mathrm{s}}\left(1-\frac{a}{H}\right)
$$

where $a$ - magnetic hardness coefficient expressed in magnetic field units. As the magnetic hardness is mainly correlated with the coercive field, which has the maximum value equal to the anisotropy field $H_{\mathrm{a}}$, therefore the relation between the anisotropy field and coefficient $a$ is assumed as $a=c H_{\mathrm{a}}$, where $c$ is a constant [15]. Consequently Eq. (9) takes the following form:

$$
[M(H)]^{2}=M_{\mathrm{s}}^{2}\left[1-\frac{2 c H_{\mathrm{a}}}{H}+\frac{\left(c H_{\mathrm{a}}\right)^{2}}{H^{2}}\right] .
$$

Assuming $x=H / \mathrm{cH}_{\mathrm{a}}$, the expansion in a series of $\ln (x)$ function was used

$$
\ln (x)=\frac{x-1}{x}+\frac{(x-1)^{2}}{2 x^{2}}+\ldots+\frac{(x-1)^{n}}{n x^{n}}+\ldots
$$

The expression (11) is valid for $x>1 / 2$. 
The coefficient $c$ in Eq. (10) estimated for Sm-Fe-N magnet with a grain diameter of $0.97 \mu \mathrm{m}$ is equal to 0.045 , and the condition $x>1 / 2$ is fulfilled. Therefore, Eq. (10) is the expansion in a series of the following formula:

$$
[M(H)]^{2} \approx-\frac{M_{\mathrm{s}}^{2}}{2}+M_{\mathrm{s}}^{2} \ln \left(\frac{H}{c H_{\mathrm{a}}}\right) .
$$

Substitution of (12) to (8) allows us to determine the rotational hysteresis energy for full range of the external magnetic field. Furthermore, the exchange energy $E_{e}$, which is the part of the total free energy was neglected, owing to its low value. Therefore, the final formula describing the rotational hysteresis energy is as follows:

$$
\begin{aligned}
\frac{W_{\mathrm{r}}}{K_{1}} & =\frac{\mu_{0} M_{\mathrm{s}} H \sin \Phi}{K_{1}} \exp \left(\frac{\mu_{0} D M_{\mathrm{s}}^{2} \ln \left(\frac{H}{c H_{\mathrm{a}}}\right)}{2 N k T}\right) \exp \left(-\frac{\mu_{0} D M_{\mathrm{s}}^{2}}{4 N k T}\right) \\
& \times \exp \left(-\frac{\mu_{0} M_{\mathrm{s}} H \cos \Phi}{N k T}\right) \int_{0}^{2 \pi} \exp \left(\frac{E_{\mathrm{a}}}{N k T}\right) \mathrm{d} \Theta .
\end{aligned}
$$

Such equation allows us to determine $A, B$, and $\beta$ coefficients occurring in (2) which are equal to

$$
A=\frac{\mu_{0} M_{\mathrm{s}} H_{\mathrm{a}}}{K_{1}} \sin \Phi_{c}-\frac{\mu_{0} D M_{\mathrm{s}}^{2}}{2 N k T} \exp \left(-\frac{\mu_{0} D M_{\mathrm{s}}^{2}}{4 N k T}\right) \int_{0}^{2 \pi} \exp \left(\frac{E_{\mathrm{a}}}{N k T}\right) \mathrm{d} \Theta
$$

$\left(\int_{0}^{2 \pi} \exp \left(\frac{E_{\mathrm{a}}}{N k T}\right) \mathrm{d} \Theta\right.$ may be numerically calculated for the examined magnet),

$$
\beta=\frac{\mu_{0} D M_{\mathrm{s}}^{2}}{2 N k T}+1
$$

and

$$
B=\frac{\mu_{0} M_{\mathrm{s}} H_{\mathrm{a}} \cos \Phi}{N k T}
$$

Dependence of $A$ and $\beta$ coefficients on $M_{\mathrm{s}}$ and demagnetization energy as well as the number of local magnetic moments $N$ was deduced from Eqs. (13) and (2). Furthermore, the value of $A$ coefficient is determined by the magnetocrystalline anisotropy constant $K_{1}$, anisotropy field $H_{\mathrm{a}}$ as well as the $\Phi$ angle. Moreover, dependence of $B$ coefficient on the anisotropy field $H_{\mathrm{a}}, \Phi$ angle, number of local magnetic moment $N$ and saturation of magnetization $M_{\mathrm{s}}$ was evidenced. However, the $B$ coefficient is not related to demagnetization field of the sample. Generally, all three coefficients, $A, B$, and $\beta$, depend on the temperature $T$.

\section{Test of rotational hysteresis energy model}

In order to verify the proposed model of rotational hysteresis energy as an example, the following calculations were performed for the $\mathrm{Sm}-\mathrm{Fe}-\mathrm{N}$ magnet with a grain diameter of $0.97 \mu \mathrm{m}$. Using formula (15) and taking the value 
TABLE

The $A, B$, and $\beta$ coefficients of Eq. (2) for Sm-Fe-N magnet with different grain diameters.

\begin{tabular}{c|r|r|c}
\hline \hline $\begin{array}{c}\text { Grain diameters } \\
D[\mu \mathrm{m}]\end{array}$ & \multicolumn{1}{|c|}{$A$} & $B$ & $\beta$ \\
\hline 8.6 & 24.83 & 8.44 & 1.69 \\
3.3 & 445.85 & 11.19 & 2.41 \\
2.2 & 956.32 & 11.75 & 2.65 \\
1.6 & 2312.66 & 11.99 & 3.01 \\
0.97 & 19991.49 & 14.56 & 3.87
\end{tabular}

of $\beta=3.87$ (Table), $T=300 \mathrm{~K}$, and $\mu_{0} M_{\mathrm{s}}=0.696 \mathrm{~T}$, the total number of local magnetic moments per volume unit $\left(1 \mathrm{~m}^{3}\right)$ was calculated for this magnet $N=1.3 \times 10^{25}$. To confirm the calculations presented above, the number of local magnetic moments was compared with the number of atoms that give the contribution to the saturation magnetization. From Mössbauer spectroscopy it results that the examined $\mathrm{Sm}-\mathrm{Fe}-\mathrm{N}$ magnet consists of three phases: $\mathrm{Sm}_{2} \mathrm{Fe}_{17} \mathrm{~N}_{0.86}$, $\mathrm{SmFe}_{5}$, and $\alpha$-Fe, therefore the contribution to the total magnetization only comes from $\mathrm{Fe}$ atoms. For $\mathrm{Sm}_{2} \mathrm{Fe}_{17} \mathrm{~N}_{x}$ compound with $x<1$, the estimated volume of the unit cell $V \cong 8.0 \times 10^{-28} \mathrm{~m}^{3}$ and the number of Fe atoms in the unit cell $n=51$. For $\mathrm{SmFe}_{5}$ phase, the number of Fe atoms in the unit cell $n=5$ and the volume of the cell $V=1.02 \times 10^{-28} \mathrm{~m}^{3}$. However, for $\alpha$-Fe phase $n=2$ and $V=0.23 \times 10^{-28} \mathrm{~m}^{3}$, respectively. Considering the volume fraction of particular magnetic phases $v_{1}=0.3$ for $\mathrm{Sm}_{2} \mathrm{Fe}_{17} \mathrm{~N}_{0.86}, \nu_{2}=0.6$ for $\mathrm{SmFe}_{5}$, and $\nu_{3}=0.1$ for $\alpha$-Fe (determined from X-ray diffraction and Mössbauer spectroscopy), the total number of Fe atoms in the volume unit $\left(1 \mathrm{~m}^{3}\right)$, giving the contribution to effective magnetization $N_{\mathrm{Fe}}=5.7 \times 10^{28}$, was calculated. This number is about three times larger than the number of local magnetic moments $N$. This is the intermediate prove that evaluated calculations are correct.

\section{Conclusions}

The proposed model of rotational hysteresis energy described by Eq. (2) gives better agreement of experimental results with the theoretical curves than the models presented in [20,21] for a wide range of the external magnetic fields. The agreement results from the introduction of $\beta$ exponent in Eq. (2) and the additional energy terms such as: demagnetization energy $E_{\mathrm{D}}$, magnetocrystalline anisotropy energy $E_{\mathrm{a}}$ as well as the exchange energy unit $E_{\mathrm{e}}$.

All the models of the magnetization reversal process are derived from the minimization of the total free energy of the magnet. However, they do not give a strong evidence, which mechanism is responsible for the magnetization reversal 
process in the magnet: nucleation of the reversed domains or pinning of domain walls. Significant factors, allowing to determine which of the magnetization reversal processes is predominant, are the microstructure properties. These properties suggest that the pinning mechanism is acting in the case of single phase magnets, and on the other hand, in the multiphase samples, the nucleation of reversed domains is predominant. The $\mathrm{Sm}^{-} \mathrm{Fe}-\mathrm{N}$ magnet studied in this paper consists of two hard magnetic phases: $\mathrm{Sm}_{2} \mathrm{Fe}_{17} \mathrm{~N}_{0.86}$ and $\mathrm{SmFe}_{5}$, as well as $\alpha$-Fe soft magnetic phase, which have a much smaller value of crystalline anisotropy energy than hard magnetic phases. Thus, the areas where $\alpha$-Fe occurs, as well as phase boundaries and grains boundaries are similar to those analyzed in Kronmüller nucleation model [25]. Therefore, there is a large probability of forming local magnetic moments that follow the above described rotational hysteresis energy model. Thus the magnetization reversal process in the examined magnets is conditioned by the nucleation of reversed domains.

\section{Acknowledgment}

The work was supported by the State Committee for Scientific Research under grant No. PBZ KBN-013 T08 42.

\section{References}

[1] J.M.D. Coey, H. Sun, J. Magn. Magn. Mater. 87, L251 (1990).

[2] K. Kobayashi, in: Proc. 13th Int. Workshop on RE Magnets and their Applications, Birmingham, September 1994, Eds. C.A.F. Manwaring, D.G.R. Jones, A.J. Williams, I.R. Harris, Birmingham 1994, p. 717.

[3] J.M.D. Coey, H. Sun, D.P.F. Hurley, J. Magn. Magn. Mater. 101, 310 (1991).

[4] M. Leonowicz, D. Januszewski, W. Kaszuwara, E. Jezierska, in: Proc. 15th Int. Workshop on RE Magnets and their Applications, Dresden, August/September 1998, Eds. L. Schultz, K.-H. Müller, Dresden 1998, p. 225.

[5] S. Brennan, X.-L. Rao, R. Skomski, N. Dempsey, J.M.D. Coey, J. Magn. Magn. Mater. 157/158, 510 (1996).

[6] P. Pawlik, J.J. Wysłocki, W. Kaszuwara, M. Leonowicz, Mater. Sci. Forum 373-376, 309 (2001).

[7] F.A.O. Cabral, S. Gama, E. de Morais, N.L. Sanjurjo, C.A. Ribeiro, C.C. Colucci, IEEE Trans. Magn. 32, 4365 (1996).

[8] K. O'Donnell, M. Aindow, I.R. Harris, R. Skomski, J.M.D. Coey, J. Magn. Magn. Mater. 157/158, 79 (1996).

[9] P. Pawlik, J.J. Wysłocki, W. Kaszuwara, M. Leonowicz, in: Proc. 15th Int. Workshop on RE Magnets and their Applications, Dresden, August/September 1998, Eds. L. Schultz, K.-H. Müller, Dresden 1998, p. 165.

[10] Jifan Hu, T. Dragon, M.-L. Sartorelli, H. Kronmüller, Phys. Status Solidi A 136, 207 (1993). 
[11] J.J. Wysłocki, J. Magn. Magn. Mater. 58, 313 (1986).

[12] J.J. Wysłocki, J. Olszewski, B. Wysłocki, S. Szymura, J. Wójcik, IEEE Trans. Magn. MAG-26, 2667 (1990).

[13] R.A. McCurrie, S. Jackson, IEEE Trans. Magn. MAG-16, 1310 (1980).

[14] J.J. Wysłocki, M. Leonowicz, H.A. Davies, IEEE Trans. Magn. MAG-29, 2806 (1993).

[15] R.M. Bozorth, Ferromagnetism, IEEE Press (Classic Reissue), New York 1993.

[16] M. Leonowicz, W. Kaszuwara, private information.

[17] E.C. Stoner, E.P. Wohlfarth, Philos. Trans. R. Soc. Lond. A 240, 599 (1948).

[18] I.S. Jacobs, C.P. Bean, Rhys. Rev. 100, 1060 (1955).

[19] S. Shtrikman, D. Treves, J. Phys. Radium 20, 286 (1959).

[20] N.L. Bryukhatov, N.A. Grintshchar, Izv. Akad. D. W. D. UdSSR Ser. Fiz. 12, 1486 (1961).

[21] L. Jahn, Hermsd. Techn. Mitt. 21, 1833 (1981).

[22] J.J. Wysłocki, P. Pawlik, W. Kaszuwara, M. Leonowicz, in: 8th European Magnetic Materials and Applications Conf., Kiev (Ukraine), September 2000, Abstracts, p. 284.

[23] N. Akulov, Ferromagnetizm, Nauka, 1939.

[24] A.H. Morrish, The Physical Principles of Magnetism, J. Wiley and Sons Inc., New York 1965.

[25] X.C. Kou, E.H.C.P. Sinnecker, R. Grössinger, G. Wiesinger, W. Rodewald, H. Kronmüller, in: Proc. 13th Int. Workshop on RE Magnets and their Applications, Birmingham, September 1994, Eds. C.A.F. Manwaring, D.G.R. Jones, A.J. Williams, I.R. Harris, Birmingham 1994, p. 753. 